\title{
Comparison of Six Supersoft X-ray Binaries
}

\author{
A.P. Cowley ${ }^{1}$ and P.C. Schmidtke ${ }^{1}$ \\ Dept. of Physics 8 Astronomy, Arizona State University, Tempe, AZ \\ 85287-1504, USA
}

\section{J.B. Hutchings ${ }^{1}$ and D. Crampton ${ }^{1}$}

Dominion Astrophysical Observatory, HIA/NRC, Victoria, B.C., V8X 4M6, Canada

\section{Introduction}

The supersoft X-ray sources are a distinct class of X-ray sources identified by $R O S A T$. They are characterized by very high luminosities $\left(\mathrm{L}_{b o l} \sim 10^{38}\right.$ ergs $\mathrm{s}^{-1}$ ) and black body temperatures of $k \mathrm{~T} \sim 30-60 \mathrm{eV}$. These sources are easily detected in the LMC and SMC because of the low column density of absorbing $\mathrm{H}$ gas. Thus, the samples found there are complete. They are much more difficult to find in the Galaxy due to soft X-ray absorption in the galactic plane.

\section{Photometry, Spectroscopy, and Models}

We have obtained multicolor photometry and spectra at CTIO of six supersoft X-ray binaries to intercompare their physical properties. These include three in the LMC (RX J0513.9-6951, CAL 83, and CAL 87), one in the SMC (SMC $13=1 \mathrm{E} 0035.4-7230$ ), and two in the Galaxy (RX J0019.8+2156 and RX J0925.7-4758).

Light curves show that their orbital periods range from $\sim 4^{h}$ to at least $\sim 1^{d}$, and they include a range of orbital inclinations (see table) from eclipsing (CAL 87) to nearly face-on (RX J0513-69, CAL 83). In addition to orbital variations, most (and perhaps all) also show long-term changes in the mean light level (e.g. CAL 83 has a range of $\sim 1$ mag (Cowley et al. 1998) and RX J0513-69 has high and low states differing by $\sim 0.8 \mathrm{mag}$ (Alcock et al. 1996)).

Spectra of all six systems show He II and O VI emission lines, and some also have phase-dependent $\mathrm{H}$ absorption. Equivalent widths of $\mathrm{He}$ II differ by $\sim 10 \times$ between systems, roughly correlated with their orbital periods (and luminosities). However, the $\mathrm{O}$ VI lines are similar in all systems suggesting they are formed in the innermost regions of the accretion disk. $\mathrm{H}$ absorption lines have negative velocities indicating gas outflow with a complex geometry.

Four of the systems show high-velocity 'jets'. In CAL 83 Crampton et al. (1987) noted long-term changes in the wings of the $\mathrm{He}$ II and $\mathrm{H} \beta$ emissions and

\footnotetext{
${ }^{1}$ Visiting Astronomers, Cerro Tololo Inter-American Observatory. CTIO is operated by AURA, Inc. under cooperative agreement with the National Science Foundation.
} 
suggested they might be due to outflowing material with velocities of $\sim 1000 \mathrm{~km}$ $\mathrm{s}^{-1}$. A 69-day period appears to fit widely scattered data from 15 years. In RX J0513-69 Crampton et al. (1996) demonstrated the presence of twin jets with outflow velocities of $\sim \pm 4000 \mathrm{~km} \mathrm{~s}^{-1}$, later confirmed by Southwell et al. (1996). Double jets with a velocity of $\sim \pm 800 \mathrm{~km} \mathrm{~s}^{-1}$ are sometimes present in RX J0019+21 (Becker et al. 1998; Cowley et al. 1998). If these are bi-polar jets, their lower velocity may be due to the fact that this system is viewed at a higher inclination angle than CAL 83 or RX J0513-69. Motch (1998) has found evidence for transient jets $\left( \pm 5200 \mathrm{~km} \mathrm{~s}^{-1}\right)$ in RX J0925-47.

Table 1. Comparison of Supersoft Sources ${ }^{a}$

\begin{tabular}{llccccccl}
\hline Name & $\begin{array}{l}\text { Per. } \\
(\mathrm{d})\end{array}$ & $\begin{array}{c}\mathrm{m}_{V} \\
(\mathrm{max})\end{array}$ & $\begin{array}{c}\Delta \mathrm{m} \\
(\mathrm{orb})\end{array}$ & $\begin{array}{c}\mathrm{K}_{H e I I} \\
\left(\mathrm{~km} \mathrm{~s}^{-1}\right)\end{array}$ & $\begin{array}{c}i \\
(\mathrm{deg})\end{array}$ & $\mathrm{M}_{V}$ & $\begin{array}{c}\mathrm{L}_{\text {bol }} / 10^{37} \\
\left(\mathrm{erg} \mathrm{s}^{-1}\right)\end{array}$ & Notes \\
\hline RX J0925 & $3.79 ?$ & 17.1 & 0.2 & 84 & $30-40$ & $\sim 0$ & $0.03-0.07$ & $5200 \mathrm{~km} \mathrm{~s}^{-1}$ jets \\
CAL 83 & 1.04 & 16.3 & 0.2 & 35 & 25 & -1.3 & $10-100$ & $1000 \mathrm{~km} \mathrm{~s}^{-1}$ jets \\
RX J0513 & 0.76 & 16.6 & 0.04 & 11 & 15 & -2.0 & $1-60$ & $4000 \mathrm{~km} \mathrm{~s}^{-1}$ jets \\
RX J0019 & 0.66 & 12.2 & 0.5 & 67 & 56 & +0.6 & $0.3-0.9$ & $800 \mathrm{~km} \mathrm{~s}^{-1}$ jets \\
CAL 87 & 0.44 & 19.0 & 1.8 & 73 & 78 & +0.3 & $\begin{array}{c}6-20 \\
\text { eclipses }\end{array}$ \\
SMC 13 & 0.17 & 20.2 & 0.3 & 100 & 75 & +1.4 & $0.8-2$ & partial eclipses \\
\hline \hline
\end{tabular}

${ }^{a}$ Data from references in text, plus Greiner (1996) and references therein

The commonly accepted model for supersoft X-ray binaries involves a white dwarf accreting matter from a $\sim 2 \mathrm{M}_{\odot}$ companion at such a high rate that steady nuclear burning occurs (van den Heuvel et al. 1992). However, mass determinations based on emission-line velocity curves show that the mass donor has only about half the mass of the compact star, making it difficult to understand what drives the mass transfer. This suggests improved spectroscopic observations and new models are needed.

We find no evidence that the Magellanic Cloud and galactic systems differ in luminosity or other parameters as a result of differences in the ages and metallicity between these galaxies.

\section{References}

Alcock, C., et al. 1996, MNRAS, 280, L49

Becker, C.M., et al. 1998, ApJ, 506, 880

Cowley, A.P., et al. 1998, ApJ, 504, 854

Crampton, D., et al. 1987, ApJ, 321, 745

Crampton, D., et al. 1996, ApJ, 456, 320

Greiner, J. 1996, Lecture Notes in Physics, 472, 299

Motch, C. 1998, A\&A, 338, L13

Southwell, K.A., et al. 1996, ApJ, 470, 1065

van den Heuvel, E.P.J., et al. 1992, A\&A, 262, 97 